\title{
Effective Computation of Maximal Sound Approximations of Description Logic Ontologies
}

\author{
Marco Console, Jose Mora, Riccardo Rosati, \\ Valerio Santarelli, and Domenico Fabio Savo \\ Dipartimento di Ingegneria Informatica Automatica e Gestionale Antonio Ruberti \\ Sapienza Università di Roma \\ \{lastname\}edis.uniroma1.it
}

\begin{abstract}
We study the problem of approximating Description Logic (DL) ontologies specified in a source language $\mathcal{L}_{S}$ in terms of a less expressive target language $\mathcal{L}_{T}$. This problem is getting very relevant in practice: e.g., approximation is often needed in ontology-based data access systems, which are able to deal with ontology languages of a limited expressiveness. We first provide a general, parametric, and semantically well-founded definition of maximal sound approximation of a DL ontology. Then, we present an algorithm that is able to effectively compute two different notions of maximal sound approximation according to the above parametric semantics when the source ontology language is OWL 2 and the target ontology language is OWL 2 QL. Finally, we experiment the above algorithm by computing the two OWL 2 QL approximations of a large set of existing OWL 2 ontologies. The experimental results allow us both to evaluate the effectiveness of the proposed notions of approximation and to compare the two different notions of approximation in real cases.
\end{abstract}

\section{Introduction}

Description Logic (DL) ontologies are the core element of ontology-based data access (OBDA) [15], in which the ontology is utilized as a conceptual view, allowing user access to the underlying data sources. In OBDA, as well as in all the current applications of ontologies requiring automated reasoning, a trade-off between the expressiveness of the ontology specification language and the complexity of reasoning in such a language must be reached. More precisely, most of the current research and development in OBDA is focusing on languages for which reasoning, and in particular query answering, is not only tractable (in data complexity) but also first-order rewritable [25]. This imposes significant limitations on the set of constructs and axioms allowed in the ontology language.

The limited expressiveness of the current ontology languages adopted in OBDA provides a strong motivation for studying the approximation of ontologies formulated in (very) expressive languages with ontologies in low-complexity languages such as OWL 2 QL. Such a motivation is not only theoretical, but also practical, given the current availability of OBDA systems and the increasing interest in applying the OBDA approach in the real world [1|6/7|16]: for instance, ontology approximation is currently 
one of the main issues in the generation of ontologies for OBDA within the use cases of the Optique EU project 1

Several approaches have recently dealt with the problem of approximating Description Logic ontologies. These can roughly be partitioned in two types: syntactic and semantic. In the former, only the syntactic form of the axioms of the original ontology is considered, thus those axioms which do not comply with the syntax of the target ontology language are disregarded [17/18]. This approach generally can be performed quickly and through simple algorithms. However, it does not, in general, guarantee soundness, i.e., to infer only correct entailments, or completeness, i.e., all entailments of the original ontology that are also expressible in the target language are preserved [14]. In the latter, the object of the approximation are the entailments of the original ontology, and the goal is to preserve as much as possible of these entailments by means of an ontology in the target language, guaranteeing soundness of the result. On the other hand, this approach often necessitates to perform complex reasoning tasks over the ontology, possibly resulting significantly slower. For these reasons, the semantic approach to ontology approximation poses a more interesting but more complex challenge.

In this paper, we study the problem of approximating DL ontologies specified in a source language $\mathcal{L}_{s}$ in terms of a less expressive target language $\mathcal{L}_{t}$. We deal with this problem by first providing a general, parametric, and semantically well-founded definition of maximal sound approximation of a DL ontology. Our semantic definition captures and generalizes previous approaches to ontology approximation [4]8 11114]. In particular, our approach builds on the preliminary work presented in [8], which proposed a similar, although non-parameterized, notion of maximal sound approximation.

Then, we present an algorithm that is able to effectively compute two different notions of maximal sound approximation according to the above parametric semantics, when the source ontology language is OWL 2 and the target ontology language is OWL 2 QL. In particular, we focus on the local semantic approximation (LSA) and the global semantic approximation (GSA) of a source ontology. These two notions of approximation correspond to the cases when the parameter of our semantics is set, respectively, to its minimum and to its maximum. Informally, the LSA of an ontology is obtained by considering (and reasoning over) one axiom $\alpha$ of the source ontology at a time, so this technique tries to approximate $\alpha$ independently of the rest of the source ontology. On the contrary, the GSA tries to approximate the source ontology by considering all its axioms (and reasoning over such axioms) at the same time. As a consequence, the GSA is potentially able to "approximate better" than the LSA, while the LSA appears in principle computationally less expensive than the GSA. Notably, in the case of OWL 2 QL, the GSA corresponds to the notion of approximation given in [14], which has been shown to be very well-suited for query answering purposes.

Finally, we experiment the above algorithm by computing the LSA and the GSA in OWL 2 QL of a large set of existing OWL 2 ontologies. The experimental results allow us both to evaluate the effectiveness of the proposed notions of approximation and to compare the two different notions of approximation in real cases. In particular, the main properties pointed out by our experimental results are the following:

${ }^{1}$ http://optique-project.eu 
1. the computation of the LSA is usually less expensive than computing the GSA of a given source ontology;

2. in many cases, both the LSA and the GSA of an ontology are very good approximations of the ontology, in the sense that the approximated ontologies actually entail a large percentage of the axioms of the source ontology;

3. in many cases, the LSA and the GSA coincide. This and the previous property imply that the computationally less expensive LSA is usually already able to compute a high-quality sound approximation of the source ontology.

The paper is structured in the following way. First, in Section 2 we recall DL ontology languages, in particular OWL 2 and OWL 2 QL. Then, in Section 3 we present our formal, parameterized notion of semantic sound approximation of an ontology, and illustrate some general properties of such a notion. In Section 4 we present the techniques for computing the GSA and the LSA of OWL 2 ontologies in OWL 2 QL. Finally, we present an experimental evaluation of the above techniques in Section 5, and draw some conclusions in Section 6.

\section{Preliminaries}

Description Logics (DLs) [3] are logics that allow one to represent the domain of interest in terms of concepts, denoting sets of objects, value-domains, denoting sets of values, attributes, denoting binary relations between objects and values, and roles denoting binary relations over objects.

In this paper we consider the DL $\mathcal{S R O I} \mathcal{Q}$ [10], which is the logic underpinning OWL 2, as the "maximal" DL considered in this paper.

Let $\Sigma$ be a signature of symbols for individual (object and value) constants and predicates, i.e., concepts, value-domains, attributes, and roles. Let $\Phi$ be the set of all $\mathcal{S R O I} \mathcal{Q}$ axioms over $\Sigma$.

An ontology over $\Sigma$ is a finite subset of $\Phi$.

A $D L$ language over $\Sigma$ (or simply language) $\mathcal{L}$ is a set of ontologies over $\Sigma$. We call $\mathcal{L}$-ontology any ontology $\mathcal{O}$ such that $\mathcal{O} \in \mathcal{L}$. Moreover, we denote by $\Phi_{\mathcal{L}}$ the set of axioms $\bigcup_{\mathcal{O} \in \mathcal{L}} \mathcal{O}$.

We call a language $\mathcal{L}$ closed if $\mathcal{L}=2^{\Phi_{\mathcal{L}}}$. As we will see in the following, there exist both closed and non-closed DL languages among the standard ones.

The semantics of an ontology is given in terms of first-order (FOL) interpretations (cf. [3]). We denote with $\operatorname{Mod}(\mathcal{O})$ the set of models of $\mathcal{O}$, i.e., the set of FOL interpretations that satisfy all the axioms in $\mathcal{O}$ (we recall that every $\mathcal{S R O} \mathcal{I} \mathcal{Q}$ axiom corresponds to a first-order sentence). As usual, an ontology $\mathcal{O}$ is said to be satisfiable if it admits at least one model, and $\mathcal{O}$ is said to entail a First-Order Logic (FOL) sentence $\alpha$, denoted $\mathcal{O}=\alpha$, if $\alpha^{\mathcal{I}}=$ true for all $\mathcal{I} \in \operatorname{Mod}(\mathcal{O})$. Moreover, given two ontologies $\mathcal{O}$ and $\mathcal{O}^{\prime}$, we say that $\mathcal{O}$ and $\mathcal{O}^{\prime}$ are logically equivalent if $\operatorname{Mod}(\mathcal{O})=\operatorname{Mod}\left(\mathcal{O}^{\prime}\right)$.

In this work we will mainly focus on two specific languages, which are OWL 2, the official ontology language of the World Wide Web Consortium (W3C) [9], and one of its profiles, OWL 2 QL [12]. Due to the limitation of space, here we do not provide a complete description of OWL 2, and refer the reader to the official W3C specification [13]. 
We now present the syntax of OWL 2 QL. We use the German notation for describing OWL 2 QL constructs and axioms, and refer the reader to [12] for the OWL functional style syntax.

Expressions in OWL 2 QL are formed according to the following syntax:
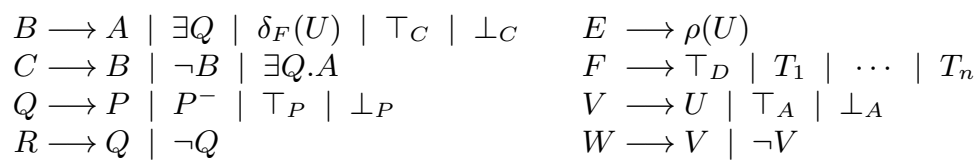

where: $A, P$, and $U$ are symbols denoting respectively an atomic concept, an atomic role, and an atomic attribute; $P^{-}$denotes the inverse of $P ; \exists Q$, also called unqualified existential role, denotes the set of objects related to some object by the role $Q ; \delta_{F}(U)$ denotes the qualified domain of $U$ with respect to a value-domain $F$, i.e., the set of objects that $U$ relates to some value in $F ; \rho(U)$ denotes the range of $U$, i.e., the set of values related to objects by $U ; T_{1}, \ldots, T_{n}$ denote $n$ unbounded value-domains (i.e., datatypes); the concept $\exists Q$.A, or qualified existential role, denotes the qualified domain of $Q$ with respect to $A$, i.e., the set of objects that $Q$ relates to some instance of $A$. $\top_{C}$, $\top_{P}, \top_{A}$, and $\top_{D}$ denote, respectively, the universal concept, role, attribute, and valuedomain, while $\perp_{C}, \perp_{P}$, and $\perp_{A}$ denote, respectively, the empty concept, role, and attribute.

An OWL 2 QL ontology $\mathcal{O}$ is a finite set of axioms of the form:

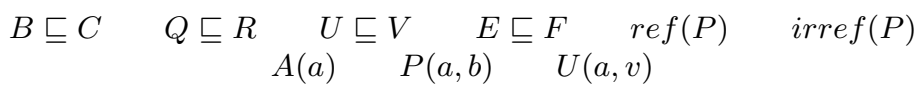

From left to right, the first four above axioms denote subsumptions between concepts, roles, attributes, and value-domains, respectively. The fifth and sixth axioms denote reflexivity and irreflexivity on roles. The last three axioms denote membership of an individual to a concept, membership of a pair of individuals to a role, and membership of a pair constituted by an individual and a value to an attribute.

From the above definition, it immediately follows that OWL 2 QL is a closed language. On the other hand, we recall that OWL 2 is not a closed language. This is due to the fact that OWL 2 imposes syntactic restrictions that concern the simultaneous presence of multiple axioms in the ontology (for instance, there exist restrictions on the usage of role names appearing in role inclusions in the presence of the role chaining constructor).

\section{Approximation}

In this section, we illustrate our notion of approximation in a target language $\mathcal{L}_{T}$ of an ontology $\mathcal{O}_{S}$ in a language $\mathcal{L}_{S}$.

Typically, when discussing approximation, one of the desirable properties is that of soundness. Roughly speaking, when the object of approximation is a set of models, this property requires that the set of models of the approximation is a superset of those of the original ontology. Another coveted characteristic of the computed ontology is that it be the "best" approximation of the original ontology. In other words, the need of keeping 
a minimal distance between the original ontology and the ontology resulting from its approximation is commonly perceived.

On the basis of these observations, the following definition of approximation in a target language $\mathcal{L}_{T}$ of a satisfiable $\mathcal{L}_{S}$-ontology is very natural.

Definition 1. Let $\mathcal{O}_{S}$ be a satisfiable $\mathcal{L}_{S}$-ontology, and let $\Sigma_{\mathcal{O}_{S}}$ be the set of predicate and constant symbols occurring in $\mathcal{O}_{S}$. An $\mathcal{L}_{T}$-ontology $\mathcal{O}_{T}$ over $\Sigma_{\mathcal{O}_{S}}$ is a global semantic approximation (GSA) in $\mathcal{L}_{T}$ of $\mathcal{O}_{S}$ if both the following statements hold:

(i) $\operatorname{Mod}\left(\mathcal{O}_{S}\right) \subseteq \operatorname{Mod}\left(\mathcal{O}_{T}\right)$;

(ii) there is no $\mathcal{L}_{T}$-ontology $\mathcal{O}^{\prime}$ over $\Sigma_{\mathcal{O}_{S}}$ such that $\operatorname{Mod}\left(\mathcal{O}_{S}\right) \subseteq \operatorname{Mod}\left(\mathcal{O}^{\prime}\right) \subset$ $\operatorname{Mod}\left(\mathcal{O}_{T}\right)$.

We denote with globalApx $\left(\mathcal{O}_{S}, \mathcal{L}_{T}\right)$ the set of all the GSAs in $\mathcal{L}_{T}$ of $\mathcal{O}_{S}$.

In the above definition, statement $(i)$ imposes the soundness of the approximation, while statement $(\mathrm{ii})$ imposes the condition of "closeness" in the choice of the approximation.

We observe that an $\mathcal{L}_{T}$-ontology which is the GSA in $\mathcal{L}_{T}$ of $\mathcal{O}_{S}$ may not exist. This is the case when, for each $\mathcal{L}_{T}$ ontology $\mathcal{O}_{T}^{\prime}$ satisfying statement $(i)$ of Definition 1 , there always exists an $\mathcal{L}_{T}$-ontology $\mathcal{O}_{T}^{\prime \prime}$ which satisfies statement $(i)$, but for which we have that $\operatorname{Mod}\left(\mathcal{O}_{S}\right) \subseteq \operatorname{Mod}\left(\mathcal{O}_{T}^{\prime \prime}\right) \subset \operatorname{Mod}\left(\mathcal{O}_{T}^{\prime}\right)$.

The following lemma provides a sufficient condition for the existence of the GSA in a language $\mathcal{L}_{T}$ of an ontology $\mathcal{O}_{S}$.

Lemma 1. Given a language $\mathcal{L}_{T}$ and a finite signature $\Sigma$, if the set of non-equivalent axioms in $\Phi_{\mathcal{L}_{T}}$ that one can generate over $\Sigma$ is finite, then for any $\mathcal{L}_{S}$-ontology $\mathcal{O}_{S}$ globalApx $\left(\mathcal{O}_{S}, \mathcal{L}_{T}\right) \neq \emptyset$.

In cases where GSAs exist, i.e., globalApx $\left(\mathcal{O}_{S}, \mathcal{L}_{T}\right) \neq \emptyset$, given two ontologies $\mathcal{O}^{\prime}$ and $\mathcal{O}^{\prime \prime}$ in globalApx $\left(\mathcal{O}_{S}, \mathcal{L}_{T}\right)$, they may be either logically equivalent or not. The condition of non-equivalence is due to the fact that the language in which the original ontology is approximated is not closed. We have the following lemma.

Lemma 2. Let $\mathcal{L}_{T}$ be a closed language, and let $\mathcal{O}_{S}$ be an ontology. For each $\mathcal{O}^{\prime}$ and $\mathcal{O}^{\prime \prime}$ belonging to globalA $x\left(\mathcal{O}_{S}, \mathcal{L}_{T}\right)$, we have that $\mathcal{O}^{\prime}$ and $\mathcal{O}^{\prime \prime}$ are logically equivalent.

Proof. Towards a contradiction, suppose that $\operatorname{Mod}\left(\mathcal{O}^{\prime}\right) \neq \operatorname{Mod}\left(\mathcal{O}^{\prime \prime}\right)$. From this, and from Definition 1 we have that $\operatorname{Mod}\left(\mathcal{O}^{\prime}\right) \not \subset \operatorname{Mod}\left(\mathcal{O}^{\prime \prime}\right)$ and $\operatorname{Mod}\left(\mathcal{O}^{\prime \prime}\right) \not \subset \operatorname{Mod}\left(\mathcal{O}^{\prime}\right)$. Hence, there exist axioms $\alpha$ and $\beta$ in $\Phi_{\mathcal{L}_{T}}$ such that $\mathcal{O}^{\prime} \models \alpha$ and $\mathcal{O}^{\prime \prime} \forall \alpha$, and $\mathcal{O}^{\prime \prime} \models \beta$ and $\mathcal{O}^{\prime} \not \models \beta$. Since both $\mathcal{O}^{\prime}$ and $\mathcal{O}^{\prime \prime}$ are sound approximations of $\mathcal{O}_{S}, \mathcal{O}_{S} \models\{\alpha, \beta\}$. Because $\mathcal{L}_{T}$ is closed, the ontology $\mathcal{O}_{\beta}^{\prime}=\mathcal{O}^{\prime} \cup\{\beta\}$ is an $\mathcal{L}_{T}$-ontology. From the above considerations it directly follows that $\operatorname{Mod}\left(\mathcal{O}_{S}\right) \subseteq \operatorname{Mod}\left(\mathcal{O}_{\beta}^{\prime}\right) \subset \operatorname{Mod}\left(\mathcal{O}^{\prime}\right)$. This means that $\mathcal{O}^{\prime}$ does not satisfy condition $(i i)$ of Definition 1 and therefore $\mathcal{O}^{\prime} \notin$ globalApx $\left(\mathcal{O}_{S}, \mathcal{L}_{T}\right)$, which is a contradiction. The same conclusion can be reached analogously for $\mathcal{O}^{\prime \prime}$. 
In other words, if the target language is closed, Lemma 2 guarantees that, up to logical equivalence, the GSA is unique.

Definition 1 is non-constructive, in the sense that it does not provide any hint as to how to compute the approximation in $\mathcal{L}_{T}$ of an ontology $\mathcal{O}_{S}$. The following theorem suggests more constructive conditions, equivalent to those in Definition 1, but first we need to introduce the notion of entailment set [14] of a satisfiable ontology with respect to a language.

Definition 2. Let $\Sigma_{\mathcal{O}}$ be the set of predicate and constant symbols occurring in $\mathcal{O}$, and let $\mathcal{L}^{\prime}$ be a language. The entailment set of $\mathcal{O}$ with respect to $\mathcal{L}^{\prime}$, denoted as $E S\left(\mathcal{O}, \mathcal{L}^{\prime}\right)$, is the set of axioms from $\Phi_{\mathcal{L}^{\prime}}$ that only contain predicates and constant symbols from $\Sigma_{\mathcal{O}}$ and that are entailed by $\mathcal{O}$.

In other words, we say that an axiom $\alpha$ belongs to the entailment set of an ontology $\mathcal{O}$ with respect to a language $\mathcal{L}^{\prime}$, if $\alpha$ is an axiom in $\Phi_{\mathcal{L}^{\prime}}$ built over the signature of $\mathcal{O}$ and for each interpretation $\mathcal{I} \in \operatorname{Mod}(\mathcal{O})$ we have that $\mathcal{I} \models \alpha$.

Clearly, given an ontology $\mathcal{O}$ and a language $\mathcal{L}^{\prime}$, the entailment set of $\mathcal{O}$ with respect to $\mathcal{L}^{\prime}$ is unique.

Theorem 1. Let $\mathcal{O}_{S}$ be a satisfiable $\mathcal{L}_{S^{-}}$-ontology and let $\mathcal{O}_{T}$ be a satisfiable $\mathcal{L}_{T^{-}}$ ontology. We have that:

(a) $\operatorname{Mod}\left(\mathcal{O}_{S}\right) \subseteq \operatorname{Mod}\left(\mathcal{O}_{T}\right)$ if and only if $E S\left(\mathcal{O}_{T}, \mathcal{L}_{T}\right) \subseteq E S\left(\mathcal{O}_{S}, \mathcal{L}_{T}\right)$;

(b) there is no $\mathcal{L}_{T}$-ontology $\mathcal{O}^{\prime}$ such that $\operatorname{Mod}\left(\mathcal{O}_{S}\right) \subseteq \operatorname{Mod}\left(\mathcal{O}^{\prime}\right) \subset \operatorname{Mod}\left(\mathcal{O}_{T}\right)$ if and only if there is no $\mathcal{L}_{T}$-ontology $\mathcal{O}^{\prime \prime}$ such that $E \bar{S}\left(\mathcal{O}_{T}, \mathcal{L}_{T}\right) \subset E S\left(\mathcal{O}^{\prime \prime}, \mathcal{L}_{T}\right) \subseteq$ $E S\left(\mathcal{O}_{S}, \mathcal{L}_{T}\right)$.

Proof. We start by focusing on the first statement. $(\Leftarrow)$ Suppose, by way of contradiction, that $\operatorname{ES}\left(\mathcal{O}_{T}, \mathcal{L}_{T}\right) \subseteq \mathrm{ES}\left(\mathcal{O}_{S}, \mathcal{L}_{T}\right)$ and that $\operatorname{Mod}\left(\mathcal{O}_{S}\right) \nsubseteq \operatorname{Mod}\left(\mathcal{O}_{T}\right)$. This means that there exists at least one interpretation that is a model for $\mathcal{O}_{S}$ but not for $\mathcal{O}_{T}$. Therefore there exists an axiom $\alpha \in \mathcal{O}_{T}$ such that $\mathcal{O}_{S} \not \neq \alpha$. Since $\mathcal{O}_{T}$ is an ontology in $\mathcal{L}_{T}$, then $\alpha$ is an axiom in $\Phi_{\mathcal{L}_{T}}$. It follows that $\alpha \in \operatorname{ES}\left(\mathcal{O}_{T}, \mathcal{L}_{T}\right)$ and that $\alpha \notin \operatorname{ES}\left(\mathcal{O}_{S}, \mathcal{L}_{T}\right)$, which leads to a contradiction.

$(\Rightarrow)$ Towards a contradiction, suppose that $\operatorname{Mod}\left(\mathcal{O}_{S}\right) \subseteq \operatorname{Mod}\left(\mathcal{O}_{T}\right)$, but $\mathrm{ES}\left(\mathcal{O}_{T}, \mathcal{L}_{T}\right) \nsubseteq \mathrm{ES}\left(\mathcal{O}_{S}, \mathcal{L}_{T}\right)$. This means that there exists at least one axiom $\alpha \in \mathrm{ES}\left(\mathcal{O}_{T}, \mathcal{L}_{T}\right)$ such that $\alpha \notin \mathrm{ES}\left(\mathcal{O}_{S}, \mathcal{L}_{T}\right)$. It follows that $\mathcal{O}_{T} \models \alpha$ while $\mathcal{O}_{S} \not \models \alpha$, which immediately implies that $\operatorname{Mod}\left(\mathcal{O}_{S}\right) \nsubseteq \operatorname{Mod}\left(\mathcal{O}_{T}\right)$. Hence we have a contradiction.

Now we prove the second statement. $(\Leftarrow)$ By contradiction, suppose that there exists an $\mathcal{L}_{T}$-ontology $\mathcal{O}^{\prime}$ such that $\operatorname{Mod}\left(\mathcal{O}_{S}\right) \subseteq \operatorname{Mod}\left(\mathcal{O}^{\prime}\right) \subset \operatorname{Mod}\left(\mathcal{O}_{T}\right)$, and that there does not exist any $\mathcal{L}_{T}$-ontology $\mathcal{O}^{\prime \prime}$ such that $\operatorname{ES}\left(\mathcal{O}_{T}, \mathcal{L}_{T}\right) \subset \mathrm{ES}\left(\mathcal{O}^{\prime \prime}, \mathcal{L}_{T}\right) \subseteq \operatorname{ES}\left(\mathcal{O}_{S}, \mathcal{L}_{T}\right)$. From what shown before, we have that $\operatorname{Mod}\left(\mathcal{O}_{S}\right) \subseteq \operatorname{Mod}\left(\mathcal{O}^{\prime}\right) \subseteq \operatorname{Mod}\left(\mathcal{O}_{T}\right)$ implies that $\mathrm{ES}\left(\mathcal{O}_{T}, \mathcal{L}_{T}\right) \subseteq \mathrm{ES}\left(\mathcal{O}^{\prime}, \mathcal{L}_{T}\right) \subseteq \mathrm{ES}\left(\mathcal{O}_{S}, \mathcal{L}_{T}\right)$. Moreover, since both $\mathcal{O}^{\prime}$ and $\mathcal{O}_{T}$ are $\mathcal{L}_{T}$ ontologies, $\operatorname{Mod}\left(\mathcal{O}^{\prime}\right) \subset \operatorname{Mod}\left(\mathcal{O}_{T}\right)$ implies that $\mathrm{ES}\left(\mathcal{O}_{T}, \mathcal{L}_{T}\right) \neq \operatorname{ES}\left(\mathcal{O}^{\prime}, \mathcal{L}_{T}\right)$. Hence, $\operatorname{ES}\left(\mathcal{O}_{T}, \mathcal{L}_{T}\right) \subset \operatorname{ES}\left(\mathcal{O}^{\prime}, \mathcal{L}_{T}\right) \subseteq \operatorname{ES}\left(\mathcal{O}_{S}, \mathcal{L}_{T}\right)$, which contradicts the hypothesis.

$(\Rightarrow)$ Suppose, by way of contradiction, that there exists an $\mathcal{L}_{T}$-ontology $\mathcal{O}^{\prime \prime}$ such that $\operatorname{ES}\left(\mathcal{O}_{T}, \mathcal{L}_{T}\right) \subset \operatorname{ES}\left(\mathcal{O}^{\prime \prime}, \mathcal{L}_{T}\right) \subseteq \operatorname{ES}\left(\mathcal{O}_{S}, \mathcal{L}_{T}\right)$ and there is no $\mathcal{L}_{T}$-ontology $\mathcal{O}^{\prime}$ 
such that $\operatorname{Mod}\left(\mathcal{O}_{S}\right) \subseteq \operatorname{Mod}\left(\mathcal{O}^{\prime}\right) \subset \operatorname{Mod}\left(\mathcal{O}_{T}\right)$. From property $(a)$ we have that $\operatorname{Mod}\left(\mathcal{O}_{S}\right) \subseteq \operatorname{Mod}\left(\mathcal{O}^{\prime \prime}\right) \subseteq \operatorname{Mod}\left(\mathcal{O}_{T}\right)$. Since both $\mathcal{O}^{\prime \prime}$ and $\mathcal{O}_{T}$ are $\mathcal{L}_{T}$ ontologies, then $\mathrm{ES}\left(\mathcal{O}_{T}, \mathcal{L}_{T}\right) \subset \mathrm{ES}\left(\mathcal{O}^{\prime \prime}, \mathcal{L}_{T}\right)$ implies that $\operatorname{Mod}\left(\mathcal{O}^{\prime \prime}\right) \neq \operatorname{Mod}\left(\mathcal{O}_{T}\right)$, which directly leads to a contradiction.

From Theorem 1 it follows that every ontology $\mathcal{O}_{T}$ which is a GSA in $\mathcal{L}_{T}$ of an ontology $\mathcal{O}_{S}$ is also an approximation in $\mathcal{L}_{T}$ of $\mathcal{O}_{S}$ according to [8], and, as we shall show in the following section, for some languages, this corresponds to the approximation in [14].

As discussed in [8], the computation of a GSA can be a very challenging task even when approximating into tractable fragments of OWL 2 [12]. This means that even though a GSA is one that best preserves the semantics of the original ontology, it currently suffers from a significant practical setback: the outcome of the computation of the approximation is tightly linked to the capabilities of the currently available reasoners for $\mathcal{L}_{S}$-ontologies. This may lead, in practice, to the impossibility of computing GSAs of very large or complex ontologies when the source language is very expressive.

We observe that the critical point behind these practical difficulties in computing a GSA of an ontology is that, in current implementations, any reasoner for $\mathcal{L}_{S}$ must reason over the ontology as a whole. From this observation, the idea for a new notion of approximation, in which we do not reason over the entire ontology but only over portions of it, arises. At the basis of this new notion, which we call k-approximation, is the idea of obtaining an approximation of the original ontology by computing the global semantic approximation of each set of $k$ axioms of the original ontology in isolation. Below we give a formal definition of the k-approximation.

In what follows, given an ontology $\mathcal{O}$ and a positive integer $k$ such that $k \leq|\mathcal{O}|$, we denote with $\operatorname{subset}_{k}(\mathcal{O})$ the set of all the sets of cardinality $k$ of axioms of $\mathcal{O}$.

Definition 3. Let $\mathcal{O}_{S}$ be a satisfiable $\mathcal{L}_{S}$-ontology and let $\Sigma_{\mathcal{O}_{S}}$ be the set of predicate and constant symbols occurring in $\mathcal{O}_{S}$. Let $\mathcal{U}_{k}=\left\{\mathcal{O}_{i}^{j} \mid \mathcal{O}_{i}^{j} \in \operatorname{globalApx}\left(\mathcal{O}_{i}, \mathcal{L}_{T}\right)\right.$, such that $\mathcal{O}_{i} \in$ subset $\left._{k}\left(\mathcal{O}_{S}\right)\right\}$. An $\mathcal{L}_{T}$-ontology $\mathcal{O}_{T}$ over $\Sigma_{\mathcal{O}_{S}}$ is a k-approximation in $\mathcal{L}_{T}$ of $\mathcal{O}_{S}$ if both the following statements hold:

- $\bigcap_{\mathcal{O}_{i}^{j} \in \mathcal{U}_{k}} \operatorname{Mod}\left(\mathcal{O}_{i}^{j}\right) \subseteq \operatorname{Mod}\left(\mathcal{O}_{T}\right)$;

- there is no $\mathcal{L}_{T}$-ontology $\mathcal{O}^{\prime}$ over $\Sigma_{\mathcal{O}_{S}}$ such that $\bigcap_{\mathcal{O}_{i}^{j} \in \mathcal{U}_{k}} \operatorname{Mod}\left(\mathcal{O}_{i}^{j}\right) \subseteq \operatorname{Mod}\left(\mathcal{O}^{\prime}\right) \subset$ $\operatorname{Mod}\left(\mathcal{O}_{T}\right)$.

The following theorem follows from Theorem 1 and provides a constructive condition for the k-approximation.

Theorem 2. Let $\mathcal{O}_{S}$ be a satisfiable $\mathcal{L}_{S}$-ontology and let $\Sigma_{\mathcal{O}_{S}}$ be the set of predicate and constant symbols occurring in $\mathcal{O}_{S}$. An $\mathcal{L}_{T}$-ontology $\mathcal{O}_{T}$ over $\Sigma_{\mathcal{O}_{S}}$ is a kapproximation in $\mathcal{L}_{T}$ of $\mathcal{O}_{S}$ if and only if:

(i) $E S\left(\mathcal{O}_{T}, \mathcal{L}_{T}\right) \subseteq E S\left(\bigcup_{\mathcal{O}_{i} \in \text { subset }_{k}\left(\mathcal{O}_{S}\right)} E S\left(\mathcal{O}_{i}, \mathcal{L}_{T}\right), \mathcal{L}_{T}\right)$;

(ii) there is no $\mathcal{L}_{T}$-ontology $\mathcal{O}^{\prime}$ over $\Sigma_{\mathcal{O}_{S}}$ such that $E S\left(\mathcal{O}_{T}, \mathcal{L}_{T}\right) \subset E S\left(\mathcal{O}^{\prime}, \mathcal{L}_{T}\right) \subseteq$ $E S\left(\bigcup_{\mathcal{O}_{i} \in \operatorname{subset}_{k}\left(\mathcal{O}_{S}\right)} E S\left(\mathcal{O}_{i}, \mathcal{L}_{T}\right), \mathcal{L}_{T}\right)$ 
Proof. (sketch) The proof can be easily adapted from the proof of Theorem 1 by observing that in order to prove the theorem one has to show that:

(a) $\bigcap_{\mathcal{O}_{i}^{j} \in \mathcal{U}_{k}} \operatorname{Mod}\left(\mathcal{O}_{i}^{j}\right) \subseteq \operatorname{Mod}\left(\mathcal{O}_{T}\right)$ if and only if $\operatorname{ES}\left(\mathcal{O}_{T}, \mathcal{L}_{T}\right) \subseteq$ $\mathrm{ES}\left(\bigcup_{\mathcal{O}_{i} \in \text { subset }_{k}\left(\mathcal{O}_{S}\right)} \mathrm{ES}\left(\mathcal{O}_{i}, \mathcal{L}_{T}\right), \mathcal{L}_{T}\right)$

(b) and that there is no $\mathcal{L}_{T}$-ontology $\mathcal{O}^{\prime}$ over $\Sigma_{\mathcal{O}_{S}}$ such that $\bigcap_{\mathcal{O}_{i}^{j} \in \mathcal{U}_{k}} \operatorname{Mod}\left(\mathcal{O}_{i}^{j}\right) \subseteq$ $\operatorname{Mod}\left(\mathcal{O}^{\prime}\right) \subset \operatorname{Mod}\left(\mathcal{O}_{T}\right)$ if and only if there is no $\mathcal{L}_{T}$-ontology $\mathcal{O}^{\prime \prime}$ over $\Sigma_{\mathcal{O}_{S}}$ such that $\mathrm{ES}\left(\mathcal{O}_{T}, \mathcal{L}_{T}\right) \subset \mathrm{ES}\left(\mathcal{O}^{\prime \prime}, \mathcal{L}_{T}\right) \subseteq \mathrm{ES}\left(\bigcup_{\mathcal{O}_{i} \in \operatorname{subset}_{k}\left(\mathcal{O}_{S}\right)} \mathrm{ES}\left(\mathcal{O}_{i}, \mathcal{L}_{T}\right), \mathcal{L}_{T}\right)$

We note that in the case in which $k=\left|\mathcal{O}_{S}\right|$, the k-approximation actually coincides with the GSA. At the other end of the spectrum, we have the case in which $k=1$. Here we are treating each axiom $\alpha$ in the original ontology in isolation, i.e., we are considering ontologies formed by a single axiom $\alpha$. We refer to this approximation as local semantic approximation (LSA).

We conclude this section with an example highlighting the difference between the GSA and the LSA.

Example 1. Consider the following OWL 2 ontology $\mathcal{O}$.

$$
\begin{aligned}
& \mathcal{O}=\{A \sqsubseteq B \sqcup C \quad B \sqsubseteq D \quad A \sqsubseteq \exists R . D \\
& B \sqcap C \sqsubseteq F \quad C \sqsubseteq D \quad \exists R . D \sqsubseteq E\} .
\end{aligned}
$$

The following ontology is a GSA in OWL 2 QL of $\mathcal{O}$.

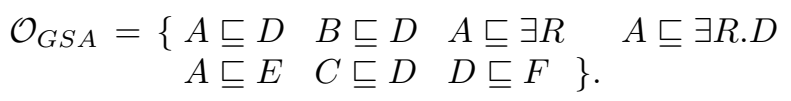

Indeed, it is possible to show that, according to Theorem 1 each axiom entailed by $\mathcal{O}_{G S A}$ is also entailed by $\mathcal{O}$, and that it is impossible to build an OWL 2 QL ontology $\mathcal{O}^{\prime}$ such that $\mathrm{ES}\left(\mathcal{O}_{G S A}, O W L 2 Q L\right) \subset \mathrm{ES}\left(\mathcal{O}^{\prime}, O W L 2 Q L\right) \subseteq \mathrm{ES}(\mathcal{O}, O W L 2 Q L)$.

Computing the LSA in OWL 2 QL of $\mathcal{O}$, i.e., its 1-approximation in OWL 2 QL, we obtain the following ontology.

$$
\begin{aligned}
& \mathcal{O}_{L S A}=\{B \sqsubseteq D \quad A \sqsubseteq \exists R \\
& C \sqsubseteq D \quad A \sqsubseteq \exists R . D\} .
\end{aligned}
$$

It is easy to see that $\operatorname{Mod}(\mathcal{O}) \subset \operatorname{Mod}\left(\mathcal{O}_{G S A}\right) \subset \operatorname{Mod}\left(\mathcal{O}_{L S A}\right)$, which means that the ontology $\mathcal{O}_{G S A}$ approximates $\mathcal{O}$ better than $\mathcal{O}_{L S A}$. This expected result is a consequence of the fact that reasoning over each single axiom in $\mathcal{O}$ in isolation does not allow for the extraction all the OWL 2 QL consequences of $\mathcal{O}$.

Moreover, from Lemma 2, it follows that every $\mathcal{O}^{\prime} \in \operatorname{globalApx}\left(\mathcal{O}_{S}, O W L 2 Q L\right)$ is logically equivalent to $\mathcal{O}_{G S A}$.

\section{Approximation in OWL 2 QL}

In this section we deal with the problem of approximating ontologies in OWL 2 with ontologies in OWL 2 QL.

Based on the characteristics of the OWL 2 QL language, we can give the following theorem. 


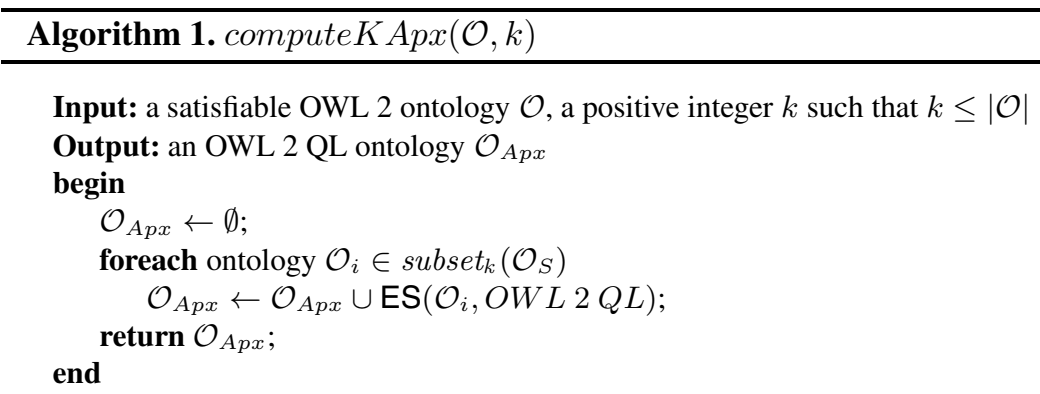

Theorem 3. Let $\mathcal{O}_{S}$ be a satisfiable OWL 2 ontology. Then the OWL 2 QL ontology $\bigcup_{\mathcal{O}_{i} \in \text { subset }_{k}\left(\mathcal{O}_{S}\right)} E S\left(\mathcal{O}_{i}, O W L 2 Q L\right)$ is the k-approximation in OWL 2 QL of $\mathcal{O}_{S}$.

Proof. (sketch) To prove the claim, we observe that Lemma 1 holds for OWL 2 QL ontologies, and this guarantees that for every OWL 2 ontology $\mathcal{O}_{S}$, there exists at least one OWL 2 QL ontology which is its GSA, i.e., globalApx $\left(\mathcal{O}_{S}, O W L 2 Q L\right) \neq \emptyset$. Moreover, we have that since OWL 2 QL is closed, for Lemma 2, all ontologies in $\mathrm{ES}\left(\mathcal{O}_{S}, O W L 2 Q L\right)$ are pairwise logically equivalent. Another consequence of the fact that OWL $2 \mathrm{QL}$ is closed is that, whichever language the original ontology $\mathcal{O}_{S}$ is expressed in, $\mathrm{ES}\left(\mathcal{O}_{S}, O W L 2 Q L\right)$ is an OWL 2 QL ontology. Furthermore, given a set of OWL 2 QL ontologies, the union of these ontologies is still an OWL 2 QL ontology. From these observations, it is easy to see that, given an OWL 2 ontology $\mathcal{O}_{S}$ and an integer $k \leq\left|\mathcal{O}_{S}\right|$, the set $\bigcup_{\mathcal{O}_{i} \in \text { subset }_{k}\left(\mathcal{O}_{S}\right)} \mathrm{ES}\left(\mathcal{O}_{i}, O W L 2 Q L\right)$ satisfies conditions $(i)$ and $(i i)$ of Theorem 2 . Hence, we have the claim.

Notably, we observe that for $k=\left|\mathcal{O}_{S}\right|$ the k-approximation $\mathcal{O}_{T}$ in OWL 2 QL of $\mathcal{O}_{S}$ is unique and coincides with its entailment set in OWL $2 \mathrm{QL}$. This means that $\mathcal{O}_{T}$ is also the approximation in OWL $2 \mathrm{QL}$ of $\mathcal{O}_{S}$ according to the notion of approximation presented in [14]. Therefore, all the properties that hold for the semantics in [14] also hold for the GSA. In particular, the evaluation of a conjunctive query $q$ without nondistinguished variables over $\mathcal{O}_{S}$ coincides with the evaluation of $q$ over $\mathcal{O}_{T}$ (Theorem 5 in [14]).

From Theorem 3 , one can easily come up with Algorithm 1 for computing the kapproximation of an $\mathcal{L}_{S}$-ontology $\mathcal{O}_{S}$ in OWL $2 \mathrm{QL}$. The algorithm first computes every subset with size $k$ of the original ontology $\mathcal{O}_{S}$. Then, it computes the ontology which is the result of the k-approximation in OWL $2 \mathrm{QL}$ of the ontology in input as the union of the entailment sets with respect to OWL 2 QL of each such subset. A naive algorithm for computing the entailment set with respect to OWL 2 QL can be easily obtained from the one given in [14] for DL-Lite languages. We can summarize it as follows. Let $\mathcal{O}$ be an ontology and let $\Sigma_{\mathcal{O}}$ be the set of predicate and constant symbols occurring in $\mathcal{O}$. The algorithm first computes the set $\Gamma$ of axioms in $\Phi_{O W L} 2 Q L$ which can be built over $\Sigma_{\mathcal{O}}$, and then, for each axiom $\alpha \in \Gamma$ such that $\mathcal{O} \models \alpha$, adds $\alpha$ to the set $\operatorname{ES}(\mathcal{O}, O W L 2 Q L)$. In practice, to check if $\mathcal{O} \models \alpha$ one can use an OWL 2 reasoner. 
Since each invocation of the OWL 2 reasoner is N2EXPTIME, the computation of the entailment set can be very costly [4].

A more efficient technique for its computation is given in [8], where the idea is to limit the number of invocations to the OWL 2 reasoner by exploiting the knowledge acquired through a preliminary exploration of the ontology. To understand the basic idea behind this technique, consider, for example, an ontology $\mathcal{O}$ that entails the inclusions $A_{1} \sqsubseteq A_{2}$ and $P_{1} \sqsubseteq P_{2}$, where $A_{1}$ and $A_{2}$ are concepts and $P_{1}$ and $P_{2}$ are roles. Exploiting these inclusions we can deduce the hierarchical structure of the general concepts that can be built on these four predicates. For instance, we know that $\exists P_{2} . A_{2} \sqsubseteq \exists P_{2}$, that $\exists P_{2} . A_{1} \sqsubseteq \exists P_{2} . A_{2}$, that $\exists P_{1} . A_{1} \sqsubseteq \exists P_{2} . A_{1}$, and so on. To obtain the entailed inclusion axioms, we begin by invoking the OWL 2 reasoner, asking for the children of the general concepts which are in the highest position in the hierarchy. So we first compute the subsumees of $\exists P_{2}$ through the OWL 2 reasoner. If there are none, we avoid invoking the reasoner asking for the subsumees of $\exists P_{2} . A_{2}$ and so on. Regarding the entailed disjointness axioms, we follow the same approach but starting from the lowest positions in the hierarchy.

The following theorem establishes correctness and termination of algorithm computeKApx.

Theorem 4. Let $\mathcal{O}_{S}$ be a satisfiable OWL 2 ontology. computeKApx $\left(\mathcal{O}_{S}, k\right)$ terminates and computes the k-approximation in $O W L 2$ QL of $\mathcal{O}_{S}$.

Proof. (sketch) Termination of compute $K A \operatorname{Apx}\left(\mathcal{O}_{S}, k\right)$ directly follows from the fact that $\mathcal{O}_{S}$ is a finite set of axioms and that, for each $\mathcal{O}_{i} \in \operatorname{subset}_{k}\left(\mathcal{O}_{S}\right)$, $\mathrm{ES}\left(\mathcal{O}_{i}, O W L 2 Q L\right)$ can be computed in finite time. The correctness of the algorithm directly follows from Theorem 3 .

\section{Experiments}

In this section we present the experimental tests that we have performed for the approximation of a suite of OWL 2 ontologies into OWL 2 QL through the two notions of approximation we have introduced earlier.

We notice that by choosing a value for $k$ different from $\left|\mathcal{O}_{S}\right|$, the computation of the entailment set becomes easier. However, observing Algorithm 1, the number of times that this step must be repeated can grow very quickly. In fact, the number of sets of axioms in $\operatorname{subset}_{k}\left(\mathcal{O}_{S}\right)$ is equal to the binomial coefficient of $\left|\mathcal{O}_{S}\right|$ over $k$, and therefore for large ontologies this number can easily become enormous, and this can be in practice a critical obstacle in the computation of the k-approximation.

For this reason, in these experiments we have focused on comparing the GSA (kapproximation with $k=\left|\mathcal{O}_{S}\right|$ ) to the LSA (k-approximation with $k=1$ ), and we reserve the study of efficient techniques for k-approximation with $1<k<\left|\mathcal{O}_{S}\right|$ for future works. Furthermore, to provide a standard baseline against which to compare the results of the GSA and LSA, we have compared both our approaches with a syntactic sound approximation approach, consisting in first normalizing the axioms in the ontology and then eliminating the ones that are not syntactically compliant with OWL 2 QL. We will refer to this approach as "SYNT". 
Table 1. Characteristics of the ontologies used in the GSA and LSA tests

\begin{tabular}{|c|c|c|c|c|c|c|}
\hline Ontology & Expressiveness & Axioms & Concepts & Roles & Attributes & OWL2 QL Axioms \\
\hline Homology & $\mathcal{A L C}$ & 83 & 66 & 0 & 0 & 83 \\
\hline Cabro & $\mathcal{A L C H \mathcal { L }}$ & 100 & 59 & 13 & 0 & 99 \\
\hline Basic vertebrate anatomy & $\mathcal{S H \mathcal { I } F}$ & 108 & 43 & 14 & 0 & 101 \\
\hline Fungal anatomy & $\mathcal{A L E \mathcal { I } +}$ & 115 & 90 & 5 & 0 & 113 \\
\hline Pmr & $\mathcal{A L U}$ & 163 & 137 & 16 & 0 & 159 \\
\hline $\mathrm{Ma}$ & $\mathcal{A L E}+$ & 168 & 166 & 8 & 0 & 167 \\
\hline General formal Ontology & $\mathcal{S H \mathcal { I }}$ & 212 & 45 & 41 & 0 & 167 \\
\hline Cog analysis & 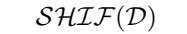 & 224 & 92 & 37 & 9 & 213 \\
\hline Time event & 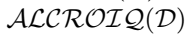 & 229 & 104 & 28 & 7 & 170 \\
\hline Spatial & $\mathcal{A L E \mathcal { H }}+$ & 246 & 136 & 49 & 0 & 155 \\
\hline Translational medicine & 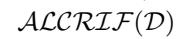 & 314 & 225 & 18 & 6 & 298 \\
\hline Biopax & $\mathcal{S H \mathcal { I N } ( \mathcal { D } )}$ & 391 & 69 & 55 & 41 & 240 \\
\hline Vertebrate skeletal anatomy & $\mathcal{A L \mathcal { E }}{ }_{+}$ & 455 & 314 & 26 & 0 & 434 \\
\hline Image & $\mathcal{S}$ & 548 & 624 & 2 & 0 & 524 \\
\hline Protein & $\mathcal{A L C F}(\mathcal{D})$ & 691 & 45 & 50 & 133 & 490 \\
\hline Pizza & $\mathcal{S H O \mathcal { N }}$ & 712 & 100 & 8 & 0 & 660 \\
\hline Ontology of physics for biology & $\mathcal{A L C H \mathcal { H }}(\mathcal{D})$ & 954 & 679 & 33 & 3 & 847 \\
\hline Plant trait & $\mathcal{A L E}+$ & 1463 & 1317 & 4 & 0 & 1461 \\
\hline Dolce & $\mathcal{S H O \mathcal { I N }}(\mathcal{D})$ & 1667 & 209 & 313 & 4 & 1445 \\
\hline Ont. of athletic events & $\mathcal{A L E \mathcal { H }}$ & 1737 & 1441 & 15 & 1 & 1722 \\
\hline Neomark & $\mathcal{A L C H} \mathcal{Q}(\mathcal{D})$ & 1755 & 55 & 105 & 488 & 842 \\
\hline Pato & $\mathcal{S H}$ & 1979 & 2378 & 36 & 0 & 1779 \\
\hline Protein Modification & $\mathcal{A L E}+$ & 1986 & 1338 & 8 & 0 & 1982 \\
\hline Po anatomy & $\mathcal{A L E}+$ & 2128 & 1294 & 11 & 0 & 2064 \\
\hline Lipid & $\mathcal{A L C H \mathcal { I N }}$ & 2375 & 716 & 46 & 0 & 2076 \\
\hline Plant & $\mathcal{S}$ & 2615 & 1644 & 16 & 0 & 2534 \\
\hline Mosquito anatomy & $\mathcal{A L E}+$ & 2733 & 1864 & 5 & 0 & 2732 \\
\hline Idomal namespace & $\mathcal{A L E R}+$ & 3467 & 2597 & 24 & 0 & 3462 \\
\hline Cognitive atlas & $\mathcal{A L C}$ & 4100 & 1701 & 6 & 0 & 3999 \\
\hline Genomic & $\mathcal{A L C Q}$ & 4322 & 2265 & 2 & 0 & 3224 \\
\hline Mosquito insecticide resistance & $\mathcal{A L E}+$ & 4413 & 4362 & 21 & 0 & 4409 \\
\hline Galen-A & 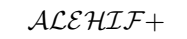 & 4979 & 2748 & 413 & 0 & 3506 \\
\hline Ni gene & $\mathcal{S H}$ & 8835 & 4835 & 8 & 0 & 8834 \\
\hline Fyp & $\mathcal{S H}$ & 15105 & 4751 & 69 & 0 & 12924 \\
\hline Fly anatomy & $\mathcal{S H}$ & 20356 & 8064 & 72 & 0 & 20353 \\
\hline EL-Galen & $\mathcal{A L E \mathcal { H } +}$ & 36547 & 23136 & 950 & 0 & 25138 \\
\hline Galen full & $\mathcal{A L \mathcal { L }} \mathcal{H} \mathcal{I} F+$ & 37696 & 23141 & 950 & 0 & 25613 \\
\hline Pr reasoned & $\mathcal{S}$ & 46929 & 35470 & 14 & 0 & 40031 \\
\hline Snomed fragment for FMA & $\mathcal{A L E \mathcal { R }}$ & 73543 & 52635 & 52 & 0 & 35004 \\
\hline Gene & $\mathcal{S H}$ & 73942 & 39160 & 12 & 0 & 73940 \\
\hline FMA OBO & $\mathcal{A L} \mathcal{E}+$ & 119560 & 75139 & 2 & 0 & 119558 \\
\hline
\end{tabular}

The suite of ontologies used during testing contains 41 ontologies and was assembled from the Bioportal ontology repository2. The ontologies that compose this suite were selected to test the scalability of our approaches both to larger ontologies and to ontologies formulated in more expressive languages. In Table 1 we present the most relevant metrics of these ontologies.

All tests were performed on a DELL Latitude E6320 notebook with Intel Core i7-2640M 2.8Ghz CPU and 4GB of RAM, running Microsoft Windows 7 Premium operating system, and Java 1.6 with $2 \mathrm{~GB}$ of heap space. Timeout was set

2 http://bioportal.bioontology.org/ 
at eight hours, and execution was aborted if the maximum available memory was exhausted. The tool used in the experiments and the suite of ontologies are available at http://diag.uniroma1.it/〜mora/ontology_approximation/ iswc2014/.

As mentioned in Section 4 the computation of the entailment set involves the use of an external OWL 2 reasoner. Therefore, the performance and the results of the computed approximations are greatly effected by the choice of the reasoner. For our tests, we have used the Pelle 3 OWL 2 reasoner (version v.2.3.0.6).

In Table 2 we present the results of the evaluation. An analysis of these results leads to the following observations.

Firstly, we were able to compute the GSA for 26 out of the 41 tested ontologies. For the remaining fifteen, this was not possible, either due to the size of the ontology, in terms of the number of its axioms, e.g., the FMA 2.0 or Gene ontologies, which have more than seventy thousand and one hundred thousand axioms, respectively, or due to its high expressivity, e.g., the Dolce ontology or the General formal ontology. The LSA approach is instead always feasible, it is quicker than the GSA approach for all but one of the tested ontologies, and it is overall very fast: no ontology took more than 250 seconds to approximate with the LSA.

Secondly, it is interesting to observe the comparison between the quality of the approximation that one can obtain through the LSA with respect to that obtained through the GSA. This relationship answers the question of whether the ontology obtained through the LSA (the "LSA ontology") is able to capture a significant portion of the one obtained through the GSA (the "GSA ontology"). Our tests in fact confirm that this is the case: out of the 26 ontologies for which we were able to compute the GSA, in only four cases the LSA ontology entails less than 60 percent of the axioms of the GSA ontology, while in twenty cases it entails more than 90 percent of them. The average percentage of axioms in the original ontologies entailed by the GSA ontologies is roughly 80 percent, and of the axioms of the GSA ontologies entailed by the LSA ontologies is roughly 87 percent.

Furthermore, the LSA provides a good approximation even for those ontologies for which the GSA is not computable. In fact, Table 3 shows the percentage of axioms of the original ontology that are entailed by the LSA ontology. Out of the twelve ontologies for which we were able to obtain this value (the remaining three ontologies caused an "out of memory" error), only in three cases it was less than 60 percent, while in four cases it was higher than 80 percent. These results are particularly interesting with respect to those ontologies for which the GSA approach is not feasible due to their complexity, as is the case for example for the Dolce ontology, for Galen-A, and for the Ontology of physics for biology. Indeed, even though these ontologies are expressed in highly expressive DL languages, the structure of the axioms that compose them is such that reasoning on each of them in isolation does not lead to much worse approximation results than reasoning on the ontology as a whole: for the nine smallest ontologies in Table 3, for which the GSA fails not because of the size of the ontology, the average percentage is 68.6.

${ }^{3}$ http://clarkparsia.com/pellet/ 
Table 2. Results of the GSA, LSA, and SYNT. The values represent, from left to right, the number of axioms in the ontology obtained through the GSA, the percentage of axioms of the original ontology that are entailed by the ontology obtained through the GSA, the number of axioms in the ontology obtained through the LSA, the percentage of axioms of the ontology obtained through the GSA that are entailed by the LSA, the number of axioms in the ontology obtained by the SYNT, the percentage of axioms of the ontology obtained through the GSA that are entailed by the ontology obtained through the SYNT, the percentage of axioms of the ontology obtained through the LSA that are entailed by the ontology obtained through the SYNT, and finally the GSA time and the LSA time (both in seconds).

\begin{tabular}{|c|c|c|c|c|c|c|c|c|c|}
\hline Ontology & $\begin{array}{c}\text { GSA } \\
\text { axioms }\end{array}$ & $\begin{array}{l}\text { GSA entails } \\
\text { original }(\%)\end{array}$ & \begin{tabular}{|c|} 
LSA \\
axioms
\end{tabular} & $\begin{array}{c}\text { LSA entails } \\
\text { GSA }(\%)\end{array}$ & \begin{tabular}{|l|} 
SYNT \\
axioms
\end{tabular} & $\begin{array}{c}\text { SYNT entails } \\
\text { GSA }(\%)\end{array}$ & $\begin{array}{c}\text { SYNT entails } \\
\text { LSA }(\%)\end{array}$ & $\begin{array}{c}\text { GSA } \\
\text { time (s) }\end{array}$ & $\begin{array}{c}\text { LSA } \\
\text { time }(\mathrm{s})\end{array}$ \\
\hline Homology & 83 & 100 & 83 & 100 & 83 & 100 & 100 & 1 & 4 \\
\hline Cabro & 233 & 96 & 121 & 100 & 100 & 100 & 100 & 4 & 2 \\
\hline Basic vertebrate anatomy & 192 & 93 & 141 & 97 & 71 & 56 & 67 & 3 & 3 \\
\hline Fungal anatomy & 318 & 98 & 140 & 69 & 113 & 69 & 100 & 2 & 2 \\
\hline Pmr & 162 & 97 & 159 & 98 & 159 & 98 & 100 & 2 & 2 \\
\hline Cog analysis & 104407 & 75 & 474 & 46 & 215 & 1 & 82 & 36 & 7 \\
\hline Time event & 93769 & 71 & 662 & 99 & 196 & 1 & 58 & 45 & 11 \\
\hline Spatial & 510 & 63 & 371 & 86 & 155 & 42 & 52 & 9 & 4 \\
\hline Translational medicine & 4089 & 86 & 505 & 99 & 275 & 30 & 64 & 19 & 7 \\
\hline Biopax & 2182057 & - & 3217 & - & 251 & - & 81 & - & 11 \\
\hline Pizza & 2587 & 91 & 755 & 92 & 678 & 92 & 99 & 7 & 4 \\
\hline Ont. of physics for biology & 1789821 & - & 1505 & - & 1241 & - & 100 & - & 7 \\
\hline Plant trait & 2370 & 99 & 1496 & 99 & 1461 & 100 & 100 & 10 & 9 \\
\hline Dolce & - & - & 2959 & - & 1555 & - & 100 & - & 8 \\
\hline Ontology of athletic events & 5073 & 99 & 2392 & 99 & 1731 & 92 & 100 & 42 & 9 \\
\hline Neomark & - & - & 39807 & - & 1723 & - & 63 & - & 50 \\
\hline Pato & 4066 & 89 & 2209 & 100 & 1976 & 78 & 99 & 99 & 18 \\
\hline Protein Modification & 2195 & 99 & 2001 & 100 & 1982 & 100 & 100 & 12 & 19 \\
\hline Po anatomy & 11486 & 96 & 2783 & 77 & 2078 & 78 & 100 & 455 & 18 \\
\hline Lipid & 14659 & 87 & 3165 & 97 & 2759 & 89 & 97 & 47 & 10 \\
\hline Plant & 18375 & 96 & 3512 & 80 & 2574 & 81 & 100 & 929 & 15 \\
\hline Ni gene & 46148 & 99 & 10415 & 90 & 8834 & 91 & 100 & 472 & 32 \\
\hline Fyp & - & - & 19675 & - & 11800 & - & 82 & - & 43 \\
\hline Fly anatomy & 460849 & 99 & 28436 & 67 & 20346 & 67 & 100 & 25499 & 45 \\
\hline EL-Galen & - & - & 70272 & - & 43804 & - & 89 & - & 59 \\
\hline Galen full & - & - & 72172 & - & 44279 & - & 89 & - & 61 \\
\hline Pr reasoned & - & - & 56085 & - & 47662 & - & 100 & - & 93 \\
\hline Snomed fragment for FMA & - & - & 140629 & - & 101860 & - & 76 & - & 250 \\
\hline Gene & - & - & 86292 & - & 73940 & - & 100 & - & 178 \\
\hline FMA OBO & - & - & 143306 & - & 119558 & - & 100 & - & 113 \\
\hline
\end{tabular}

Finally, both the GSA and LSA compare favorably against the syntactic sound approximation approach. In fact, the average percentage of axioms in the LSA and GSA ontologies that are entailed by the ontologies obtained through the SYNT approach are respectively roughly 90 percent and 72 percent. While the latter result is to be expected, the former is quite significant, even more so when one considers that the LSA is very 
Table 3. LSA results for ontologies for which the GSA is not computable

\begin{tabular}{|c|c|c|c|c|}
\hline Ontology & $\begin{array}{c}\text { Original } \\
\text { axioms }\end{array}$ & $\begin{array}{c}\text { LSA } \\
\text { axioms }\end{array}$ & $\begin{array}{c}\text { LSA entails } \\
\text { original (\%) }\end{array}$ & $\begin{array}{c}\text { LSA } \\
\text { time (s) }\end{array}$ \\
\hline General formal ontology & 212 & 264 & 67 & 6 \\
Biopax & 391 & 3204 & 53 & 11 \\
Protein & 691 & 10720 & 47 & 20 \\
Ontology of physics for biology & 954 & 1074 & 75 & 7 \\
Dolce & 1667 & 2914 & 78 & 8 \\
Neomark & 1755 & 38966 & 46 & 50 \\
Genomic & 4322 & 9844 & 65 & 54 \\
Galen-A & 4979 & 8568 & 70 & 26 \\
Fyp & 15105 & 19672 & 85 & 43 \\
EL-Galen & 36547 & 70272 & - & 59 \\
Galen full & 37696 & 72172 & - & 61 \\
Pr reasoned & 46929 & 55391 & 83 & 93 \\
Gene & 73543 & 140629 & - & 250 \\
FMA OBO & 73942 & 86289 & 99 & 178 \\
SNOMED fragment for FMA & 119560 & 143306 & 99 & 113 \\
\hline
\end{tabular}

fast. Indeed, a "gain" of 10 percent of axiom entailments by the LSA with respect to the SYNT in the case of large ontologies such as Galen and Snomed translates to tens of thousands of preserved axioms in very little computation time.

In conclusion, the results gathered from these tests corroborate the usefulness of both the global semantic approximation and the local semantic approximation approaches. The former provides a maximal sound approximation in the target language of the original approach, and is in practice computable in a reasonable amount of time for the majority of the tested ontologies. The latter instead represents a less optimal but still very effective solution for those ontologies for which the GSA approach goes beyond the capabilities of the currently-available ontology reasoners.

\section{Conclusions}

In this paper we have addressed the problem of ontology approximation in Description Logics and OWL, presenting (i) a parameterized semantics for computing sound approximations of ontologies, (ii) algorithms for the computation of approximations (the GSA and the LSA) of OWL 2 ontologies in OWL 2 QL, and (iii) an extensive experimental evaluation of the above techniques, which empirically proves the validity of our approach.

The present work can be extended in several ways. First, while we have focused on sound approximations, it would be interesting to also consider complete approximations of ontologies. Also, we would like to study the development of techniques for $k$-approximations different from GSA and LSA, i.e., for $k$ such that $1<k<\left|\mathcal{O}_{S}\right|$, as well as to analyze the possibility of integrating ontology module extraction techniques in our approach. Then, this work has not addressed the case when there are differences 
in the semantic assumptions between the source and the target ontology languages. For instance, differently from OWL 2 and its profiles, some DLs (e.g., DL-Lite $A$ [15]) adopt the Unique Name Assumption (UNA). This makes our approach not directly applicable, for instance, if we consider OWL 2 as the source language and DL-Lite $A$ as the target language. The reason is that the UNA implies some axioms (inequalities between individuals) that can be expressed in OWL 2 but cannot be expressed in DL-Lite $A$. We aim at extending our approach to deal with the presence of such semantic discrepancies in the ontology languages. Finally, we are very interested in generalizing our approach to a full-fledged ontology-based data access scenario [15], in which data sources are connected through declarative mappings to the ontology. In that context, it might be interesting to use both the ontology and the mappings in the target OBDA specification to approximate a given ontology in the source OBDA specification.

Acknowledgments. This research has been partially supported by the EU under FP7 project Optique (grant n. FP7-318338).

\section{References}

1. Antonioli, N., Castanø, F., Civili, C., Coletta, S., Grossi, S., Lembo, D., Lenzerini, M., Poggi, A., Savo, D.F., Virardi, E.: Ontology-based data access: the experience at the Italian Department of Treasury. In: Proc. of the Industrial Track of CAiSE 2013. CEUR, vol. 1017, pp. 9-16 (2013), Ceur-ws.org

2. Artale, A., Calvanese, D., Kontchakov, R., Zakharyaschev, M.: The DL-Lite family and relations. J. of Artificial Intelligence Research 36, 1-69 (2009)

3. Baader, F., Calvanese, D., McGuinness, D., Nardi, D., Patel-Schneider, P.F. (eds.): The Description Logic Handbook: Theory, Implementation and Applications. Cambridge University Press (2003)

4. Botoeva, E., Calvanese, D., Rodriguez-Muro, M.: Expressive approximations in DL-Lite ontologies. In: Dicheva, D., Dochev, D. (eds.) AIMSA 2010. LNCS (LNAI), vol. 6304, pp. 21-31. Springer, Heidelberg (2010)

5. Calvanese, D., De Giacomo, G., Lembo, D., Lenzerini, M., Rosati, R.: Tractable reasoning and efficient query answering in description logics: The DL-Lite family. J. of Automated Reasoning 39(3), 385-429 (2007)

6. Calvanese, D., et al.: Optique: OBDA Solution for Big Data. In: Cimiano, P., Fernández, M., Lopez, V., Schlobach, S., Völker, J. (eds.) ESWC 2013. LNCS, vol. 7955, pp. 293-295. Springer, Heidelberg (2013)

7. Civili, C., Console, M., De Giacomo, G., Lembo, D., Lenzerini, M., Lepore, L., Mancini, R., Poggi, A., Rosati, R., Ruzzi, M., Santarelli, V., Savo, D.F.: MASTRO STUDIO: Managing ontology-based data access applications. PVLDB 6, 1314-1317 (2013)

8. Console, M., Santarelli, V., Savo, D.F.: Efficient approximation in DL-Lite of OWL 2 ontologies. In: Proc. of DL 2013. CEUR Workshop Proceedings, vol. 1014, pp. 132-143. ceurws.org (2013)

9. Hitzler, P., Krötzsch, M., Parsia, B., Patel-Schneider, P.F., Rudolph, S.: OWL 2 Web Ontology Language: Primer. W3C Recommendation (2012), http: / /www.w3 .org/TR/2012/REC-ow12-primer-20121211/

10. Horrocks, I., Kutz, O., Sattler, U.: The even more irresistible $\mathcal{S R O} \mathcal{I} \mathcal{Q}$. In: Proc. of KR 2006, pp. 57-67 (2006) 
11. Lutz, C., Seylan, I., Wolter, F.: An Automata-Theoretic Approach to Uniform Interpolation and Approximation in the Description Logic EL. In: Proc. of KR 2012. AAAI Press (2012)

12. Motik, B., Grau, B.C., Horrocks, I., Wu, Z., Fokoue, A., Lutz, C.: Owl 2 web ontology language: Profiles, 2nd edn. W3C Recommendation (2012),

http://www.w3.org/TR/ow12-profiles/

13. Motik, B., Parsia, B., Patel-Schneider, P.F.: OWL 2 Web Ontology Language Structural Specification and Functional-Style Syntax. W3C Recommendation (2012), http://www.w3.org/TR/2012/REC-ow12-syntax-20121211/

14. Pan, J.Z., Thomas, E.: Approximating OWL-DL ontologies. In: Proc. of AAAI 2007, pp. 1434-1439 (2007)

15. Poggi, A., Lembo, D., Calvanese, D., De Giacomo, G., Lenzerini, M., Rosati, R.: Linking data to ontologies. In: Spaccapietra, S. (ed.) Journal on Data Semantics X. LNCS, vol. 4900, pp. 133-173. Springer, Heidelberg (2008)

16. Rodríguez-Muro, M., Kontchakov, R., Zakharyaschev, M.: Ontology-based data access: Ontop of databases. In: Alani, H., et al. (eds.) ISWC 2013, Part I. LNCS, vol. 8218, pp. 558-573. Springer, Heidelberg (2013)

17. Tserendorj, T., Rudolph, S., Krötzsch, M., Hitzler, P.: Approximate OWL-reasoning with Screech. In: Calvanese, D., Lausen, G. (eds.) RR 2008. LNCS, vol. 5341, pp. 165-180. Springer, Heidelberg (2008)

18. Wache, H., Groot, P., Stuckenschmidt, H.: Scalable instance retrieval for the semantic web by approximation. In: Dean, M., Guo, Y., Jun, W., Kaschek, R., Krishnaswamy, S., Pan, Z., Sheng, Q.Z. (eds.) WISE 2005 Workshops. LNCS, vol. 3807, pp. 245-254. Springer, Heidelberg (2005) 